\title{
Gestational Weight Gain in Obese Patients and Adverse Pregnancy Events
}

\author{
Shelly H. Tien ${ }^{*}$, Dana Villines ${ }^{2}$, Barbara V. Parilla ${ }^{3}$ \\ ${ }^{1}$ Department of Obstetrics and Gynecology, Advocate Illinois Masonic Medical Center, Chicago, USA \\ ${ }^{2}$ Department of Research, Advocate Illinois Masonic Medical Center, Chicago, USA \\ ${ }^{3}$ Department of Obstetrics and Gynecology, Division of Maternal-Fetal Medicine, Advocate Illinois Masonic \\ Medical Center, Chicago, USA \\ Email: shtien@gmail.com, tienx015@umn.edu
}

Received 27 April 2014; revised 1 June 2014; accepted 8 June 2014

Copyright (C) 2014 by authors and Scientific Research Publishing Inc.

This work is licensed under the Creative Commons Attribution International License (CC BY). http://creativecommons.org/licenses/by/4.0/

(c) (i) Open Access

\section{Abstract}

Objectives: To examine pre-pregnancy obesity and gestational weight gain as predictors for adverse pregnancy outcomes in a predominantly non-white obstetric resident clinic population. Methods: Prenatal charts for patients with pre-pregnancy obesity cared for at our resident clinic from January 1, 2008 through December 31, 2010 were reviewed. Adverse maternal outcomes were grouped into a "Composite Morbidity Index" (CMI-M) and included gestational diabetes, gestational hypertension, preeclampsia, superimposed preeclampsia, dystocia, operative delivery, Cesarean section for arrest disorders, wound infection and disruption, and thromboembolic events. Fetal events, similarly categorized into a composite adverse fetal index (CMI-F), included macrosomia, Apgar at 5 minutes ( $\leq 3)$, NICU admission, congenital anomalies and intrauterine fetal demise. Results: 627 women with a singleton pregnancy and a pre-pregnancy body mass index (BMI) of 30 and greater were included in the analysis. As measured by the composite morbidity index, women with Class III obesity at their first prenatal visit were more likely to have at least one or more maternal and fetal complications compared to women with Class II or Class I obesity. For adverse maternal outcomes (CMI-M), 40.2\%, 33.8\%, and $27.4 \%$ of women within each respective obesity class experienced an adverse event $(p=0.027)$. Applying the CMI-F, fetal complications were observed in $28.2 \%, 18 \%$, and $13.9 \%$ of Class III, II, and I obesity $(p=0.003)$. Total gestational weight gain per week was significantly greater for patients with one or more maternal complications $(p=0.045)$. Conclusion: Among an obese, resident clinic population comprised primarily of women of ethnic minorities, pre-pregnancy body mass index was the strongest indicator for adverse maternal and fetal outcomes.

\section{Keywords}

Gestational Weight Gain, Obesity and Pregnancy, Obesity and Adverse Pregnancy Outcomes

\footnotetext{
${ }^{*}$ Corresponding author.
} 


\section{Introduction}

Domestic and worldwide, obesity is a public health issue that continues to be a complex, growing dilemma. According to recent NHANES data, approximately $68.8 \%$ of the US population is overweight or obese, with $35.7 \%$ having a body mass index (BMI) of at least 30. Among adult women of reproductive age 20 and greater, 64.5\% are overweight or obese [1]. As obesity affects most every aspect of the population, more women of reproductive age are beginning their prenatal care obese. The antepartum, intrapartum, and postpartum complications of obesity are broad and well-delineated. In the antepartum period, obesity greatly increases the likelihood for the development of gestational diabetes [2]-[4] (OR 7.7 - 11) [5] and hypertensive disorders (OR 3 - 4.4) [5], as is demonstrable in a dose-response relationship. Obese parturients are more likely to have obstructive sleep apnea, which is associated with preeclampsia (OR 3.55) [6], a heighted risk for cesarean delivery (OR 8.1), and indicated preterm birth (OR 2.6) [7].

Obese patients are at increased risk of having offspring with congenital anomalies, and nearly two fold as likely to have an intrauterine fetal demise [8]-[11]. In addition to adverse antepartum and fetal events, obese women are at heightened risk for intrapartum complications such as dysfunctional labor, macrosomia, dystocia, operative delivery, and Cesarean section [12] [13].These women are more likely to develop postoperative infections (OR 1.6) [12] and thromboembolic complications (OR 1.93) [12].

In a large cohort of healthy women followed for a median of 4.5 years after childbirth, pre-pregnant obesity was strongly associated with the development of myocardial infarction or stroke [HR 2.63 (95\% CI, 1.41 - 4.91) and HR 1.89 (95\% CI, 1.25 - 2.84)], respectively, compared to normal weight women [14]. Increasing evidence suggests that children born to obese mothers are themselves at increased risk for developing obesity, metabolic syndrome, and diabetes later in life [15] [16].

In conjunction with pre-pregnancy, obesity is the compelling issue of gestational weight gain. Women who gain excessive weight during pregnancy are at heightened risk for adverse events. Hedderson et al. demonstrated that compared with a weight gain of $<0.60 \mathrm{lb} / \mathrm{wk}$, a rate of weight gain of $0.60-0.88 \mathrm{lb} / \mathrm{wk}$ was associated with an odds ratio of 1.43 for gestational diabetes [3]. When the rate of weight gain was $0.89 \mathrm{lb} /$ wk or more, this increased to an odds ratio of 1.74 [3].

Our resident patient population consists primarily of women from minority ethnic groups many of whom are overweight or obese prior to conception. This study aims to examine the associations between pre-pregnancy body mass index, gestational weight gain, and the subsequent development of maternal and fetal adverse events in an obese resident clinic population. We anticipate that our findings will parallel what has been described in the literature. We are interested in more closely examining adverse health events in a predominantly non-white, pre-pregnant obese population, and comparing outcomes within varying classes of obesity.

\section{Material and Methods}

The OB/GYN Treatment Center at our institution is an independent resident clinic, where the patients are cared for by obstetrics and gynecology residents under supervision from faculty. A retrospective chart review of patients was performed with IRB approval. We approached the billing department at the clinic and all patients who had a prenatal visit from January 1, 2008 through December 31, 2010 were identified through ICD-9 codes. Each patient's electronic medical record was reviewed, which involved scanned prenatal records, hospital progress notes, and delivery records. Paper charts were reviewed if electronic medical records were found to be incomplete. BMI was calculated from self-reported pre-pregnancy weight and height, or earliest recorded weight. Among patients with a greater than 5lb discordance between self-reported pre-pregnancy weight and documented weight at first prenatal visit, the first prenatal weight was used if the patient presented to care prior to 12 weeks of gestation. Patients were included in the analysis if they had a pre-pregnancy or earliest recorded BMI of 30 or greater and a singleton gestation. Patients were excluded if they had a pre-pregnancy BMI less than 30, multiple gestations, an elective abortion or miscarriage, or were lost to follow up and/or did not deliver at our institution. Patients who filled inclusion criteria but had two or more deliveries during the time frame were also analyzed. Charts from 2981 patients were reviewed. 572 patients who fulfilled criteria had one delivery during the time frame. 55 patients fulfilled criteria and had 2 or more deliveries during the time frame and their first delivery was used for analysis.

\section{Weight Variables}

BMI at delivery was calculated using height and documented weight at hospital admission. As patients pre- 
sented at various gestational ages for their first prenatal visit, the total number of prenatal visits was collected, and the gestational weight gain (GWG) in pounds per week was calculated to standardize this variable. For the purpose of analysis, the patients were divided into 3 classes of obesity: Class I (BMI 30-34.9), Class II (BMI 35 39.9), and Class III (BMI 40 and greater).

\section{Composite Morbidity Index}

Information on adverse maternal and fetal events was collected: gestational diabetes, gestational hypertension, preeclampsia, superimposed preeclampsia, dystocia, operative delivery, macrosomia (defined as weight $>4000$ g), Cesarean section for arrest disorders, wound infection, wound disruption, thromboembolic events, Apgar at 5 minutes, NICU admission, congenital anomalies, and intrauterine fetal demise (IUFD).

The adverse maternal and fetal events were grouped into a Composite Morbidity Index (CMI) for maternal events (CMI-M) and for fetal events (CMI-F). Maternal events included gestational diabetes, gestational hypertension, preeclampsia, superimposed preeclampsia, dystocia, operative delivery, Cesarean section for arrest disorders, wound infection, wound disruption, and thromboembolic events. Fetal events included macrosomia, Apgar at 5 minutes $(\leq 3)$, NICU admission, congenital anomalies and IUFD. CMI-F could not be calculated for 38 subjects (6\%) due to missing data. An analysis was performed to evaluate the risk for each composite index for each class of obesity. Patients were characterized as having an adverse event if they had at least one complication per index category. Patients who maintained or lost weight during their pregnancy were also evaluated. After the analysis, the variable of macrosomia was removed from the composite morbidity index, as there were no patients who had an infant with a weight of greater than $4000 \mathrm{~g}$.

\section{Demographic Variables}

Information was collected on pre-existing diabetes and chronic hypertension. Patients were classified as pregestational diabetics if they had documented Type I or Type II diabetes (DM) prior to pregnancy, or if they were diagnosed with diabetes in pregnancy prior to 24 weeks of gestational age. Patients were identified as having chronic hypertension (HTN) if they had hypertension (systolic blood pressures of $140 \mathrm{~mm} \mathrm{Hg}$ or greater, diastolic blood pressures of $90 \mathrm{~mm} \mathrm{Hg}$ or greater, or both) before pregnancy, prior to 20 weeks, or required antihypertensive medication prior to pregnancy. Demographic information was collected on ethnicity, gravity, parity, and age.

\section{Statistical Analysis}

Data analysis was performed using SPSS $18^{\circledR}$ for Windows (SPSS Inc., Chicago, IL) statistical software. Analysis was performed using Chi-square for group comparison with dichotomous data, t-tests and analysis of variance (ANOVA) for group comparison (obesity class and ethnicity) of continuous data. Pearson's correlation coefficient was used to determine the association between total GWG and the number of complications for each index. Logistic regression analysis was performed for univariate and multivariate models to examine the association between CMI-M and CMI-F as the dependent variables and obesity class at first PNV, weekly GWG, the total number of prenatal visits (PNV), maternal age, parity, as well as pre-gestation variables of diabetes and hypertension as predictor variables. Statistical significance was determined at $\mathrm{p} \leq 0.05$ for all analyses.

\section{Results}

\subsection{Demographic Characteristics}

Table 1 displays the demographic characteristics of the sample. Frequencies and percentages for each complication are displayed in Table 2. The mean number of complications for the total sample was $0.82(\mathrm{SD}=1.09)$ with $0.38(\mathrm{SD}=0.62)$ mean number of maternal complications and $0.20(\mathrm{SD}=0.46)$ mean number of fetal complications. The most frequently reported maternal complication was gestational DM (10.5\%) and the most frequently reported fetal complication was NICU admission (15.9\%) (Table 2).

\subsection{Weight Changes and Morbidity Index}

Weekly weight gain (in pounds) did not differ by obesity class $(\mathrm{p}=0.276)$, but BMI at delivery did differ between the three groups $(\mathrm{p}<0.001)$. Subjects in Class III obesity at first PNV were more likely than subjects in- 
Table 1. Demographic characteristics.

\begin{tabular}{|c|c|}
\hline Demographics & Total Sample \\
\hline \multicolumn{2}{|l|}{ Ethnicity } \\
\hline Caucasian & $85(13.6 \%)$ \\
\hline African American & $74(11.8 \%)$ \\
\hline Hispanic & $435(69.4 \%)$ \\
\hline Other/Unknown & $33(5.2 \%)$ \\
\hline \multicolumn{2}{|c|}{ Number of deliveries in time frame of interest } \\
\hline Single & $572(91.2 \%)$ \\
\hline Multiple & $55(8.8 \%)$ \\
\hline Age [mean (SD)] & $29.14(6.15)$ \\
\hline Weekly GWG (lbs) [mean (SD)] & $1.81(2.53)$ \\
\hline BMI at first PNV [mean (SD)] & $36.15(5.93)$ \\
\hline BMI at delivery [mean (SD)] & $39.45(6.55)$ \\
\hline \multicolumn{2}{|l|}{ BMI at first PNV } \\
\hline Class I & 339 (54.1\%) \\
\hline Class II & $160(25.5 \%)$ \\
\hline Class III & $122(19.5 \%)$ \\
\hline Unknown & $6(1.0 \%)$ \\
\hline \multicolumn{2}{|l|}{ BMI at delivery } \\
\hline$<30$ & $6(1.0 \%)$ \\
\hline Class I & $150(23.9 \%)$ \\
\hline Class II & $237(37.8 \%)$ \\
\hline Class III & $224(35.7 \%)$ \\
\hline Unknown & $10(1.6 \%)$ \\
\hline \multicolumn{2}{|l|}{ Parity } \\
\hline Nulliparous & $116(18.5 \%)$ \\
\hline Multiparous & 456 (72.7\%) \\
\hline
\end{tabular}

Table 2. Morbidity index.

\begin{tabular}{cc}
\hline Composite morbidity index-maternal & Total sample \\
\hline No complications & $429(68.4 \%)$ \\
Has 1 or more complications & $198(31.6 \%)$ \\
Number of complications [mean (SD)] & $0.38(0.62)$ \\
Variables in the CMI-Maternal & \\
Gestational DM & $66(10.5 \%)$ \\
Gestational HTN & $19(3.0 \%)$ \\
Preeclampsia & $30(4.8 \%)$ \\
Superimposed preeclampsia & $23(3.7 \%)$ \\
Dystocia & $17(2.7 \%)$ \\
Operative delivery & $4(0.6 \%)$ \\
Cesarean section for arrest disorders & $60(9.6 \%)$ \\
Wound infection & $11(1.8 \%)$ \\
Wound disruption & $5(0.8 \%)$ \\
Thromboembolic events & $3(0.5 \%)$ \\
\hline Composite Morbidity Index-Fetal & Total Sample \\
No complications & $484(77.2 \%)$ \\
Has 1 or more complications & $105(16.7 \%)$ \\
number of complications [mean (SD)] & $0.20(0.46)$ \\
Variables in the CMI-Fetal & \\
NICU admission & $100(15.9 \%)$ \\
Apgar at 5 minutes ( $\leq 3)$ & $4(0.7 \%)$ \\
Congenital anomalies & $20(3.2 \%)$ \\
Intrauterine fetal demise & $3(0.5 \%)$ \\
\hline
\end{tabular}

\footnotetext{
${ }^{*}$ CMI-F could not be calculated for 38 subjects (6.1\%) due to missing data.
} 
Class II or Class I obesity to have one or more complications as measured by the CMI-M and CMI-F: $40.2 \%$, $33.8 \%$, and $27.4 \%$ respectively for CMI-M ( $\mathrm{p}=0.027)$ and $28.2 \%, 18 \%$, and $13.9 \%$ respectively for CMI-F $(\mathrm{p}=$ 0.003) (Table 3). Additionally, the mean number of complications for maternal and fetal was highest in Class III (CMI-M: $\mathrm{x}=0.50, \mathrm{SD}=0.70$; CMI-F: $\mathrm{x}=0.35, \mathrm{SD}=0.64)$ and Class II was higher (CMI-M: $\mathrm{x}=0.43, \mathrm{SD}=$ 0.67; CMI-F: $\mathrm{x}=0.19, \mathrm{SD}=0.41$ ) than Class I (CMI-M: $\mathrm{x}=0.32$, SD $=0.55$; CMI-F: $\mathrm{x}=0.15, \mathrm{SD}=0.39$ ) $(\mathrm{CMI}-\mathrm{M} \mathrm{p}=0.010$; CMI-F $\mathrm{p}<0.001)$. The total GWG per week was higher for subjects with one or more complications on the CMI-M ( $\mathrm{p}=0.045)$ but not for the CMI-F $(\mathrm{p}=0.772)$ in comparison to subjects with no complications (Figure 1).

Fifty-five subjects (8.8\%) in the sample did not gain weight during their pregnancy with six subjects maintaining their exact weight and forty-nine subjects losing weight. The average weight loss was 0.95 pounds (SD = 1.43). Of the subjects who lost weight, there were no statistically significant differences in the CMI-F and CMI-M ( $\mathrm{p}=0.105$ and 0.400 ). In the CMI-F group, $75.5 \%$ had no fetal complications and $24.5 \%$ had one or more fetal complications. In the CMI-M group, $72.7 \%$ had no maternal complications and $23.7 \%$ had one or more maternal complications (data not shown).

\subsection{Multivariate Analysis of CMI Predictors}

BMI at first PNV, weekly GWG, the total number of PNV, maternal age, parity, pre-gestation DM and pre-gestation HTN were evaluated as predictors of complications as measured by the CMI-M (Table 4) and CMI-F (Table 5). By univariate analysis, BMI at first PNV, pre-gestation HTN, weekly GWG, the total number of PNV and parity were associated with having one or more complications on the CMI-M (p-values range from $<0.001$ to $\mathrm{p}=0.038$ ). When all significant univariate predictors were entered into the multivariate model, only parity remained as a statistically significant predictor of maternal complications $(\mathrm{OR}=0.39, \mathrm{CI}=0.25-0.61, \mathrm{p}<$ $0.001)$.

Three predictors were associated with the fetal index by univariate analysis. BMI at first PNV, pre-gestation $\mathrm{DM}$ and the total number of PNV were associated with having one or more complications on the CMI-F (pvalues range from 0.001 to 0.011 ). All three predictors remained statistically significant in the multivariate analysis, with total number of prenatal visits appearing to have a protective effect [BMI at first PNV (OR $=1.05$, $\mathrm{CI}=1.01-1.09, \mathrm{p}=0.005)$; pre-gestation $\mathrm{DM}(\mathrm{OR}=2.41, \mathrm{CI}=1.37-4.26, \mathrm{p}=0.002)$; total number of PNV $(\mathrm{OR}=0.88, \mathrm{CI}=0.82-0.95, \mathrm{p}=0.001)]$.

\subsection{Ethnicity}

Table 6 examines weight variables and CMI by ethnicity. Mean BMI at delivery was highest for African American subjects $(x=42.71, \mathrm{SD}=7.53)$ although the difference was only statistically significant in comparison to Hispanic subjects $(\mathrm{p}<0.001)$. Caucasian $(\mathrm{x}=40.50, \mathrm{SD}=7.14)$ and Hispanic $(\mathrm{x}=38.81, \mathrm{SD}=6.08)$ subjects' $\mathrm{BMI}$ at delivery did not differ statistically. Similarly, African American subjects had the highest mean number of complications on the CMI-M ( $\mathrm{x}=0.43, \mathrm{SD}=0.69)$ and CMI-F $(\mathrm{x}=0.36, \mathrm{SD}=0.60)$ but the difference was only statistically significant in comparison to Caucasian $(x=0.20, \mathrm{SD}=0.43)$ and Hispanic subjects $(\mathrm{x}=0.17$, $\mathrm{SD}=0.43)$ for the CMI-F.

\section{Discussion}

Among our clinic resident population of obese patients, pre-pregnancy BMI was the greatest indicator of adverse outcomes. Compared to Class I obese patients, Class II and Class III obese patients were more likely to have complications, and the likelihood of complications was directly correlated with increasing pre-pregnancy BMI. Total GWG per week was higher for subjects with one or more complications on the CMI-M ( $p=0.045)$ but not for the CMI-F $(\mathrm{p}=0.772)$ in comparison to subjects with no complications.

The most frequently reported maternal adverse events were gestational DM (10.5\%) and Cesarean section for arrest disorders (9.6\%). The most frequently reported fetal complication was NICU admission (15.9\%). After controlling for preexisting maternal medical disease, in the multivariate analysis, earliest recorded BMI was still significantly associated with adverse fetal outcomes. These findings are consistent with the literature which demonstrates that pre-pregnancy obesity is a more powerful marker for adverse outcomes. 
Table 3. Weight variables and composite morbidity index by obesity class.

\begin{tabular}{ccccc}
\hline & Class I obesity & Class II obesity & Class III obesity & p-Value \\
\hline Weekly GWG (lbs) [mean (SD)] & $1.98(2.45)$ & $1.68(2.51)$ & $1.60(2.75)$ & 0.276 \\
BMI at delivery [mean (SD)] & $35.91(4.48)$ & $40.05(3.28)$ & $48.46(5.72)$ & 0.000 \\
Composite morbidity index-maternal & & & & \\
No complications & $246(72.6 \%)$ & $106(66.3 \%)$ & $73(17.2 \%)$ & 0.027 \\
Has 1 or more complications & $93(27.4 \%)$ & $54(33.8 \%)$ & $49(40.2 \%)$ & 0.010 \\
Number of complications [mean (SD)] & $0.32(0.55)$ & $0.43(0.67)$ & $0.50(0.70)$ & \\
Composite morbidity index-fetal & & & & 0.003 \\
No complications & $278(86.1 \%)$ & $123(82.0 \%)$ & $79(71.8 \%)$ & $31(28.2 \%)$ \\
Has 1 or more complications & $45(13.9 \%)$ & $27(18.0 \%)$ & $0.35(0.64)$ & 0.000 \\
\hline Number of complications [mean (SD)] & $0.15(0.39)$ & $0.19(0.41)$ & & \\
\hline
\end{tabular}

Table 4. Univariate and multivariate predictors of composite morbidity index-Maternal.

\begin{tabular}{|c|c|c|c|c|c|}
\hline & \multicolumn{2}{|c|}{ Univariate } & \multicolumn{3}{|c|}{ Multivariate } \\
\hline & Odds ratio & p-Value & Odds ratio & $95 \% \mathrm{CI}$ & p-Value \\
\hline BMI at first PNV & 1.03 & 0.038 & 1.02 & $0.99-1.06$ & 0.165 \\
\hline Pre-gestation DM & 0.88 & 0.571 & - & - & - \\
\hline Pre-gestation hypertension & 1.77 & 0.006 & 1.52 & $0.94-2.44$ & 0.086 \\
\hline Weekly GWG (lbs) & 1.07 & 0.034 & 1.06 & $0.99-1.14$ & 0.107 \\
\hline Total PNV & 1.07 & 0.013 & 1.06 & $0.99-1.12$ & 0.094 \\
\hline Maternal age & 1.00 & 0.913 & - & - & - \\
\hline Parity & 0.36 & 0.000 & 0.39 & $0.25-0.61$ & 0.000 \\
\hline
\end{tabular}

Table 5. Univariate and multivariate predictors of composite morbidity index-Fetal.

\begin{tabular}{|c|c|c|c|c|c|}
\hline & \multicolumn{2}{|c|}{ Univariate } & \multicolumn{3}{|c|}{ Multivariate } \\
\hline & Odds ratio & p-Value & Odds ratio & $95 \% \mathrm{CI}$ & p-Value \\
\hline BMI at first PNV & 1.06 & 0.001 & 1.05 & $1.01-1.09$ & 0.005 \\
\hline Pre-gestation DM & 1.95 & 0.011 & 2.41 & $1.37-4.26$ & 0.002 \\
\hline Pre-gestation hypertension & 1.35 & 0.240 & - & - & - \\
\hline Weekly GWG (lbs) & 0.99 & 0.772 & - & - & - \\
\hline Total PNV & 0.90 & 0.006 & 0.88 & $0.82-0.95$ & 0.001 \\
\hline Maternal age & 1.01 & 0.453 & - & - & - \\
\hline Parity & 0.68 & 0.151 & - & - & - \\
\hline
\end{tabular}

In the multivariate analysis for maternal outcomes, only parity remained statistically significant for adverse maternal outcomes. This may be an effect of the particular components of the maternal composite morbidity index. For example, the variables of gestational diabetes and gestational hypertension would not apply to patients with preexisting diabetes and hypertension. Though these women may be more likely to develop superimposed preeclampsia, the numbers may have been too small. In addition, the study only examined one delivery among patients in the specified time frame. Among the small group of women who had more than one delivery during the time frame $(n=55)$, only the first delivery was used for analysis. It is possible that multiparous women were more likely to be heavier with each subsequent pregnancy, and therefore more likely to have poor outcomes. Parity may have been a marker for increased weight gain for women.

Our study evaluated women who were predominantly of a minority ethnic group. Nearly $70 \%$ of our sample size included women who self-identified as Hispanic. 59.3\% of Hispanic women were Class I obese at their first PNV, compared to $35.1 \%$ of African-American, and $42.9 \%$ of Caucasian. $16 \%$ of Hispanic women, over onethird (36.5\%) of African-American women, and 27.4\% of Caucasian women had a BMI of 40 or greater at their first prenatal visit. 
Table 6. Weight variables and composite morbidity index by ethnicity.

\begin{tabular}{|c|c|c|c|c|}
\hline & Caucasian & African American & Hispanic & p-Value \\
\hline Weekly GWG [mean (SD)] & $2.07(2.74)$ & $2.03(2.93)$ & $1.75(2.45)$ & 0.491 \\
\hline BMI at delivery [mean (SD)] & $40.50(7.14)$ & $42.71(7.53)$ & $38.81(6.08)$ & 0.000 \\
\hline \multicolumn{5}{|l|}{ CMI-Maternal } \\
\hline No complications & $56(65.9 \%)$ & $46(62.2 \%)$ & 304 (69.9\%) & \multirow{2}{*}{0.364} \\
\hline Has 1 or more complications & $29(34.1 \%)$ & $28(37.8 \%)$ & $131(30.1 \%)$ & \\
\hline Number of complications [mean (SD)] & $0.35(0.50)$ & $0.43(0.69)$ & $0.37(0.62)$ & 0.377 \\
\hline \multicolumn{5}{|l|}{ CMI-Fetal } \\
\hline No complications & $65(81.3 \%)$ & 47 (69.1\%) & $346(84.6 \%)$ & \multirow{2}{*}{0.008} \\
\hline Has 1 or more complications & $15(18.7 \%)$ & $21(30.9 \%)$ & $63(15.4 \%)$ & \\
\hline Number of complications [mean (SD)] & $0.20(0.43)$ & $0.36(0.60)$ & $0.17(0.43)$ & 0.005 \\
\hline \multicolumn{5}{|l|}{ BMI at first PNV } \\
\hline Class I obesity & $36(42.9 \%)$ & $26(35.1 \%)$ & $255(59.3 \%)$ & \multirow{3}{*}{0.000} \\
\hline Class II obesity & $25(29.8 \%)$ & $21(28.4 \%)$ & $106(24.7 \%)$ & \\
\hline Class III obesity & $23(27.4 \%)$ & 27 (36.5\%) & $69(16.0 \%)$ & \\
\hline
\end{tabular}

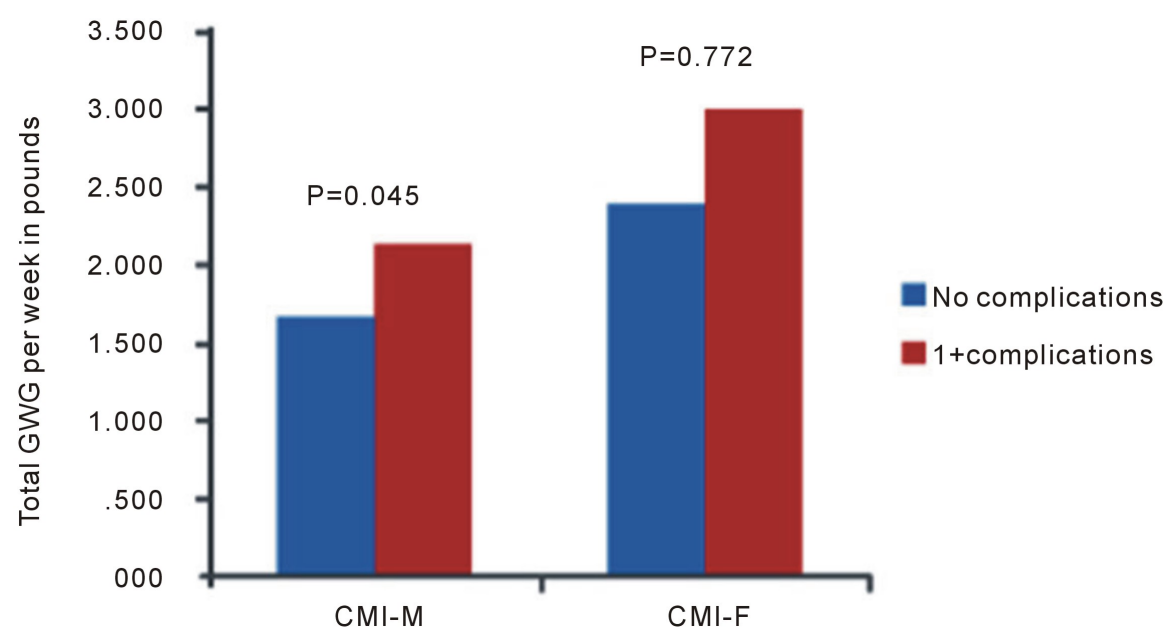

Figure 1. Total GWG per week (in pounds) for subjects with and without complications as measured by the CMI-M and CMI-F.

The high rates of obesity are consistent with national data that demonstrate a greater incidence of overweight and obesity in minority populations. From 2009-2010, 36.3\% of all US women age 20 and greater were obese. When stratified by ethnicity, $33.4 \%$ of white women, $58.6 \%$ of Non-Hispanic black, and $44.3 \%$ of Mexican American women were obese [1]. 18.3\% of all women had a BMI of $35-39.9$, and $8.2 \%$ of all women had a BMI of at least 40 . Among Non-Hispanic black women, the percentages are notably increased, at $30.9 \%$ and $18 \%$, respectively [1].

As the group with the highest BMI, 37.8\% of African American women had one or more maternal complication, and 30.9\% of African American women had one or more fetal complication, the greatest prevalence of adverse outcomes of the three ethnic groups. Weekly gestational weight gain did not differ between the ethnic groups.

Weaknesses of this study include the component of self reported pre-pregnancy weight, as well as the limitations inherent to a retrospective review. Though our patients were weighed at every prenatal visit, those who presented late to prenatal care may not have had an accurate recollection of pre-pregnancy weight. Strengths include our study population, mostly women of Non-Caucasian ethnic groups. Our patients were predominately of minority ethnic groups (94.8\%), with nearly $70 \%$ of Hispanic origin. Literature examining health-related associations of Non-Caucasian populations tends to be sparse. 
A small proportion of our patients maintained their weight or loss an average of $0.95 \mathrm{lb}$ during their care. There was no significant difference in the maternal or fetal composite morbidity index. These numbers were small and the study was not designed specifically to examine weight maintenance or weight loss, and improved outcomes. This could be an area of further investigation, especially in ethnic minority populations.

\section{Conclusions}

Optimizing weight prior to conception appears to be crucial for improved pregnancy outcomes. The strongest and most consistent predictor for adverse pregnancy events was directly related to earliest recorded BMI. In clinical practice, it can be challenging to provide pre-conception nutrition and weight loss counseling as many patients do not establish medical care until they have achieved pregnancy. For women who do present for preventive gynecologic care, providers have an opportunity to provide basic health maintenance education on nutrition, exercise, and smoking cessation. They also have the opportunity to inquire about plans for future pregnancy, and educate patients about optimizing pre-pregnancy weight. It may be beneficial to utilize additional resources and direct patients to weight loss programs and nutrition counseling as an integral part of routine health maintenance. In our specific population of minority ethnic women, many of these interventions may need to be tailored and implemented with a mindfulness of cultural differences. Nutrition counseling should involve healthful options that are realistic choices within a patient's dietary repertoire. Exercise, though an essential component to weight loss and health, can be challenging to implement and maintain. Many patients have multiple work and child-care responsibilities, financial barriers, and may lack safe space for outdoor exercise. Exploring realistic options for physical activity for patients appears to be a crucial component of health maintenance.

For a predominantly non-white, resident clinic population, pre-pregnancy and earliest BMI was the greatest predictor of adverse pregnancy outcomes. Efforts need to be focused on helping these women achieve a healthy weight prior to conception, especially in minority populations with higher rates of pre-pregnant obesity.

\section{References}

[1] Flegal, K.M., Carroll, M.D., Kit, B.K. and Ogden, C.L. (2012) Prevalence of Obesity and Trends in the Distribution of Body Mass Index among US Adults, 1999-2010. JAMA, 307, 491-497. http://dx.doi.org/10.1001/jama.2012.39

[2] Weiss, J.L., Malone, F.D., Emig, D., Ball, R.H., Nyberg, D.A., Comstock, C.H., Saade, G., Eddleman, K., Carter, S.M., Craigo, S.D., Carr, S.R. and D’Alton, M.E. (2004) Obesity, Obstetric Complications and Cesarean Delivery Rate: A Population Based Screening Study. American Journal of Obstetrics \& Gynecology, 190, 1091-1097. http://dx.doi.org/10.1016/j.ajog.2003.09.058

[3] Hedderson, M.M., Gunderson, E.P. and Ferrara, A. (2010) Gestational Weight Gain and Risk of Gestational Diabetes Mellitus. Obstetrics \& Gynecology, 115, 597-604. http://dx.doi.org/10.1097/AOG.0b013e3181cfce4f

[4] Whiteman, V.E., Rao, K., Duan, J., Alio, A., Marty, P. and Salihu, H. (2009) Interval Body Mass Index (BMI) Changes Influence the Occurrence of Pre-Eclampsia and Diabetes in Subsequent Pregnancy. American Journal of Obstetrics \& Gynecology, 201, S227. http://dx.doi.org/10.1016/j.ajog.2009.10.483

[5] Ovesen, P., Rasmussen, S. and Kesmodel, U. (2011) Effect of Prepregnancy Maternal Overweight and Obesity on Pregnancy Outcome. Obstetrics \& Gynecology, 118, 305-312. http://dx.doi.org/10.1097/AOG.0b013e3182245d49

[6] Louis, J., Auckley, D., Miladinovic, B., Shepherd, A., Mencin, P., Kumar, D., Mercer, B. and Redline, S. (2012) Perinatal Outcomes Associated with Obstructive Sleep Apnea in Obese Pregnant Women. Obstetrics \& Gynecology, 120, 1085-1092.

[7] Louis, J.M., Auckley, D., Sokol, R.J. and Mercer, B.M. (2010) Maternal and Neonatal Morbidities Associated with Obstructive Sleep Apnea Complicating Pregnancy. American Journal of Obstetrics \& Gynecology, 202, 261.e1-5.

[8] Chu, S.Y., Kim, S.Y., Lau, J., Schmid, C.H., Dietz, P.M., Callaghan, W.M. and Curtis, K.M. (2007) Maternal Obesity and Risk of Stillbirth: A Metaanalysis. American Journal of Obstetrics \& Gynecology, 197, 223-228. http://dx.doi.org/10.1016/j.ajog.2007.03.027

[9] Stothard, K.J., Tennant, P.W., Bell, R. and Rankin, J. (2009) Maternal Overweight and Obesity and the Risk of Congenital Anomalies: A Systematic Review and Meta-Analysis. JAMA, 301, 636-650. http://dx.doi.org/10.1001/jama.2009.113

[10] Rasmussen, S.A., Chu, S.Y., Kim, S.Y., Schmid, C.H. and Lau, J. (2008) Maternalobesity and Risk of Neural Tube Defects: A Metaanalysis. American Journal of Obstetrics \& Gynecology, 198, 611-619. http://dx.doi.org/10.1016/j.ajog.2008.04.021

[11] Whiteman, V.E., Crisan, L., Duan, J., Alio, A., Marty, P. and Salihu, H. (2009) The Impact of Inter-Pregnancy Body 
Mass Index (BMI) Changes on Risk of Intrauterine Fetal Demise (IUFD). American Journal of Obstetrics \& Gynecology, 201, S226-S227. http://dx.doi.org/10.1016/j.ajog.2009.10.482

[12] Robinson, H.E., O’Connell, C.M., Joseph, K.S. and McLeod, N.L. (2006) Maternal Outcomes in Pregnancies Complicated by Obesity. Obstetrics \& Gynecology, 106, 1357-1364. http://dx.doi.org/10.1097/01.AOG.0000188387.88032.41

[13] Vahratian, A., Zhang, J., Troendle, J.F., Savitz, D.A. and Siega-Riz, A.M. (2004) Maternal Prepregnancy Overweight and Obesity and the Pattern of Labor Progression in Term Nulliparous Women. Obstetrics \& Gynecology, 104, 943951. http://dx.doi.org/10.1097/01.AOG.0000142713.53197.91

[14] Schmiegelow, M.D., Andersson, C., Køber, L., Andersen, S.S., Olesen, J.B., Jensen, T.B., Azimi, A., Nielsen, M.B., Gislason, G. and Torp-Pedersen, C. (2014) Prepregnancy Obesity and Associations with Stroke and Myocardial Infarction in Women in the Years after Childbirth: A Nationwide Cohort Study. Circulation, 129, 330-337. http://dx.doi.org/10.1161/CIRCULATIONAHA.113.003142

[15] Hamilton, J.K., Odrobina, E., Yin, J., Hanley, A.J., Zinman, B. and Retnakaran, R. (2010) Maternal Insulin Sensitivity during Pregnancy Predicts Infant Weight Gain and Adiposity at 1 Year of Age. Obesity, 18, 340-346. http://dx.doi.org/10.1038/oby.2009.231

[16] Institute of Medicine. Weight Gain during Pregnancy: Reexamining the Guidelines. Report Brief May 2009. National Academy of Sciences, Washington DC. 
Scientific Research Publishing (SCIRP) is one of the largest Open Access journal publishers. It is currently publishing more than 200 open access, online, peer-reviewed journals covering a wide range of academic disciplines. SCIRP serves the worldwide academic communities and contributes to the progress and application of science with its publication.

Other selected journals from SCIRP are listed as below. Submit your manuscript to us via either submit@scirp.org or Online Submission Portal.
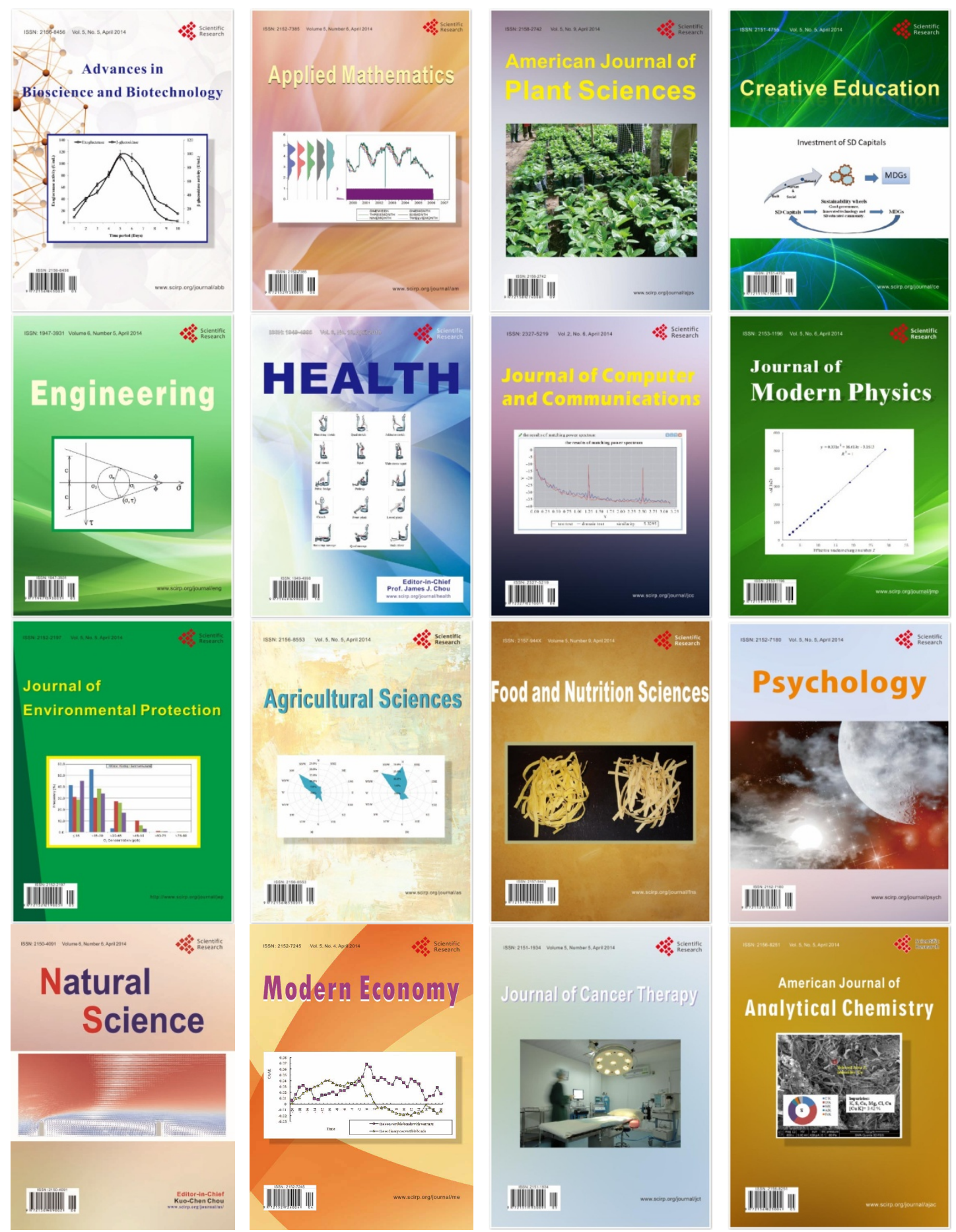